\title{
MultiKOC: Multi-One-Class Classifier Based K-Means Clustering
}

\author{
Loai Abdallah ${ }^{1, *}$, Murad Badarna ${ }^{1,2}$, Waleed Khalifa ${ }^{3}$ and Malik Yousef 4 (D) \\ 1 Department of Information Systems, Yezreel Valley Academic College, Emek Yezreel 1930600, Israel; \\ muradb@yvc.ac.il \\ 2 Department of Information Systems, University of Haifa, Haifa 3498838, Israel \\ 3 Department of Computer Science, The College of Sakhnin, Sakhnin 3081003, Israel; Khwalid@sakhnin.ac.il \\ 4 Department of Information Systems, Zefat Academic College, Safed 1320611, Israel; malik.yousef@gmail.com \\ * Correspondence: loaia@yvc.ac.il; Tel.: +972-(50)-5714178
}

Citation: Abdallah, L.; Badarna, M.; Khalifa, W.; Yousef, M. MultiKOC: Multi-One-Class Classifier Based K-Means Clustering. Algorithms 2021, 14, 134. https://doi.org/10.3390/ a14050134

Academic Editors: Frank Werner and Davide Verzotto

Received: 26 February 2021

Accepted: 21 April 2021

Published: 23 April 2021

Publisher's Note: MDPI stays neutral with regard to jurisdictional claims in published maps and institutional affiliations.

Copyright: (c) 2021 by the authors. Licensee MDPI, Basel, Switzerland. This article is an open access article distributed under the terms and conditions of the Creative Commons Attribution (CC BY) license (https:/ / creativecommons.org/licenses/by/ $4.0 /)$.

\begin{abstract}
In the computational biology community there are many biological cases that are considered as multi-one-class classification problems. Examples include the classification of multiple tumor types, protein fold recognition and the molecular classification of multiple cancer types. In all of these cases the real world appropriately characterized negative cases or outliers are impractical to achieve and the positive cases might consist of different clusters, which in turn might lead to accuracy degradation. In this paper we present a novel algorithm named MultiKOC multi-one-class classifiers based K-means to deal with this problem. The main idea is to execute a clustering algorithm over the positive samples to capture the hidden subdata of the given positive data, and then building up a one-class classifier for every cluster member's examples separately: in other word, train the OC classifier on each piece of subdata. For a given new sample, the generated classifiers are applied. If it is rejected by all of those classifiers, the given sample is considered as a negative sample, otherwise it is a positive sample. The results of MultiKOC are compared with the traditional one-class, multione-class, ensemble one-classes and two-class methods, yielding a significant improvement over the one-class and like the two-class performance.
\end{abstract}

Keywords: one-class; clustering based classification; K-means

\section{Introduction}

The aim of the one-class classification model is to distinguish data belonging to the target class from other possible classes [1-5]. This is an interesting problem because there are many real-world situations where a representative set of labeled examples for the second class is difficult to obtain or not available at all. This situation occurs in many real-word problems, such as in medical diagnosis for breast cancer detection [6,7], in the prediction of protein-protein interactions [8], the one-class recognition of cognitive brain functions [3], text mining [9], functional Magnetic Resonance Imaging [10], signature verification [11], biometrics [12] and bioinformatics [5,13-16], and social media [17].

In the literature, a vast amount of research has been carried out to tackle the problem of how to implement a multi-class classifier by an ensemble of one-class classifiers [18,19]. Lai et al. [20] proposed a method for combining different one-class classifiers for the problem of image retrieval. They reported that combining multi-SVM-based classifiers improves the retrieval precision. In a similar way, Tax et al. [21] suggest combining different oneclass classifiers to improve the performance and the robustness of the classification for the handwritten digit recognition problem.

A multi-one-class SVMs technique (OC-SVM) that combines a beforehand clustering process for detecting hidden messages in digital images was provided by Lyu et al. [22]. They showed that a multi-one-class SVM significantly simplifies the training stage of the classifiers and that even though the overall detection improves with an increasing number of hyperspheres, the false-positive rate increases considerably when the number 
of the hyperspheres increases. Menahem et al. [23] suggested a different multiple oneclass classification approach called TUPSO, which combines multi-one-class classifiers via a metaclassifier. They showed that TUPSO outperforms existing methods such as the OC-SVM. Ban et al. [24] proposed multiple one-class classifiers to deal with the nonlinear classification and the feature space problem. The multiple one-class classifiers were trained on each class in order to extract a decision function based on minimum distance rules. This proposed method outperforms the OC-SVM, as shown in their experiments.

In the domain of the computational biology community much work exists on multiple one-class classification. A multi-one-class classification approach to detect novelty in gene expression data was proposed by Spinosa et al. [25]. The approach combined different one-class classifiers such as the OC-KNN and OC-Kmeans. For a given sample, the final classification is considered by the majority votes of all classifiers. It was shown that the robustness of the classification was increased because each classifier judges the sample from a different point-of-view. For the avian influenza outbreak classification problem, a similar approach was provided by Zhang et al. [26].

In classification we assume that two-class data consist of two pure compact clusters of data, but in many cases one of the clusters might consist of multiple subclusters. For a certain dataset, a special method is required and the one-class reveals insufficient results. In this paper we propose a new approach called MultiKOC (Multi-one-class classifier based on K-means) which is an ensemble of one-class classifiers that, as a first step, devises the positive class into clusters or-subdata using the K-means applied to the examples of the data (not on the features space) and in the second step it trains a one-class classifier for each cluster (subdata). The main idea of our approach is to execute the K-means clustering algorithm over the positive examples. Next, a one-class classifier for each cluster is constructed separately. For a given new sample, our algorithm applies all the generated one-class classifiers. If it is classified as positive by at least one of those classifiers then it will be considered as a positive sample, otherwise it is considered as a negative sample. In our experiments we show that the proposed approach outperforms the one-class. In addition, we show that MultiKOC is stable over a different number of clusters.

The most significant contributions of our research are:

1. The proposed new approach in the way that it first clusters the positive data into clusters that each cluster form a subdata, before the classification process.

2. The suggested preprocessing method (i.e., the clustering phase) prevents the drawback of using only a single hypersphere generated by the one-class classifier which may not provide a particularly compact support for the training data.

3. Experimental results showing that our new approach significantly improves the accuracy of the classification against other OC classifieres.

The rest of this paper is organized as follows: Section 2 describes the necessary preliminaries. Our MultiKOC approach is described in Section 3 and evaluated in Section 4. Our main discussions and future work can be found in Section 5.

\section{Preliminaries}

\subsection{One-Class Methods}

In general, a binary learning (two-class) approach to a given data discovery considers both positive and negative classes by providing examples from the two-classes to a learning algorithm in order to build a classifier that will attempt to discriminate between them. The most common term for this kind of learning is supervised learning where the labels of the two-classes are known beforehand and are provided by the teacher (supervisor).

One-class uses only the information for the target class (positive class) to build a classifier which is able to recognize the examples belonging to its target and reject others as outliers. Among the many classification algorithms available, we chose four one-class algorithms to compare their one-class and two-class versions with our suggested tool. We give a brief description of different one-class classifiers and we refer to the references $[27,28]$ for additional details including a description of the parameters and thresholds. The 
LIBSVM library [29] was used as implementation of the OC-SVM (one-class using the RBF kernel function). The WEKA software [30] that is integrated in Knime [31] was used for the one and two-class classifiers.

\subsection{One-Class Support Vector Machines (OC-SVM)}

Support Vector Machines (SVMs) are a learning machine developed as a two-class approach [32,33]. The use of one-class SVM was originally suggested by [28]. One-class SVM is an algorithmic method that produces a prediction function trained to "capture" most of the training data. For that purpose, a kernel function is used to map the data into a feature space where the SVM is employed to find the hyperplane with maximum margin from the origin of the feature space. In this use, the margin to be maximized between the two classes (in two-class SVM) becomes the distance between the origin and the support vectors which define the boundaries of the surrounding circle, (or hypersphere in high-dimensional space) which encloses the single class. The study of [34] presents a multi-class classifier based on weighted one-class support vector machines (OCSVM) operating in the clustered feature space reporting very interesting results.

\subsection{One-Class Classifiers}

Hempstalk et al. [35] have developed many one-class classifiers that rely on the simple idea of using the standard two-class learning algorithm by combining density and class probability estimation. They have used the reference distribution to generate artificial data to be used as the negative examples. In other word, the two-class algorithm requires both positive and negative data. We assume that the positive data are given so one need to generate artificial negative data to be subject to the two-class classifier. The idea suggested by them actually allows to convert each two-class to one-class classifiers by generating the artificial negative data.

The one-class classification, by combining density and class probability estimation, was implemented on WEKA. We have considered the related node in Knime called OneClassClassifier (version 3.7) in order to examine different OC classifiers. We have considered J48, random forest, Naïve Bayes and SVM.

\section{MultiKOC-Multi-One-Class Classifiers}

As described in the previous methods, the classifier will be trained on a positive class. However, in real-world data, the positive class might consist of different subsets (see Figure 1). The classic multi-one-class classifiers use the positive samples in order to train different classifiers, then they run the ensemble classification for new instances. As a result, if we train the classifier over all the points from those subsets then the negative class will be a part of this training procedure, yielding low performance.

The main problem with this technique (i.e., classic multi-one-class) is that the oneclass classifiers do not see the negative samples (Blue points in Figure 1). As a result, the classifier will classify those points (blue points) as a positive class. To overcome this issue, we decided to train one-classifier for each subset and instead to execute one-classifier we apply multi-one-class classifiers using only one subset. For a given new instance all the one-class classifiers are employed, where if at least one of them assigns it to the positive class then it will be considered as a positive. Otherwise, it will be considered as a negative.

The main challenge of this technique is to identify the subsets. For instance, in Figure 1 we aim to identify the pink, green, black, and red subsets. Based on the fact that the points belonging to the same subset are more similar than the samples from different subsets we decided to use clustering techniques to identify the different subsets as illustrated in Figure 2. It is important to note that here: (1) we cluster only the positive class into several clusters and (2) based on our empirical experiments we see that the number of clusters is not critical. Moreover, considering two different subsets as a one subset is a more problematic situation than splitting one subset into two subsets. 


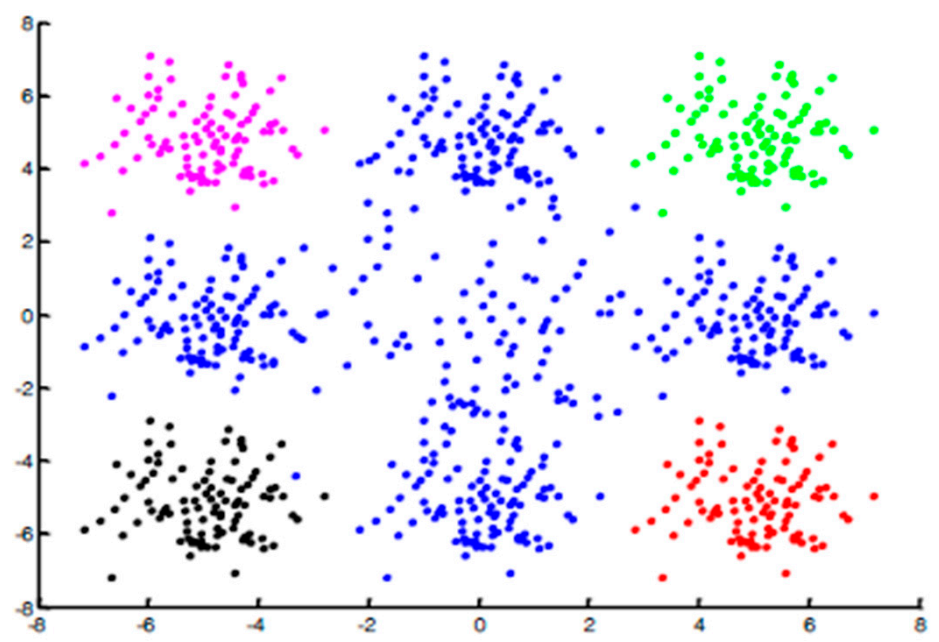

Figure 1. The positive class consists of four subgroups. The negative class is in blue color. Each cluster has a different color (pink, green, black, and red).

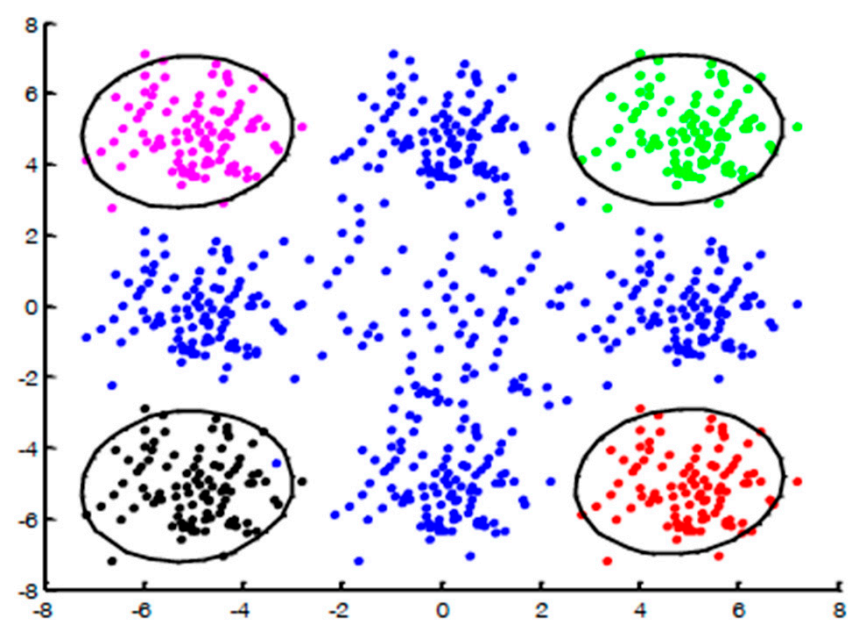

Figure 2. The MultiKOC trained over the positive examples. As can be seen, the positive examples are classified into four different clusters.

To alleviate this type of data we propose the MultiKOC Classifier that works subset of the positive data. Our approach trains the one-class classifier on each subset of the positive class detected by the clustering algorithm K-means (see Figure 3) as following Algorithm 1:

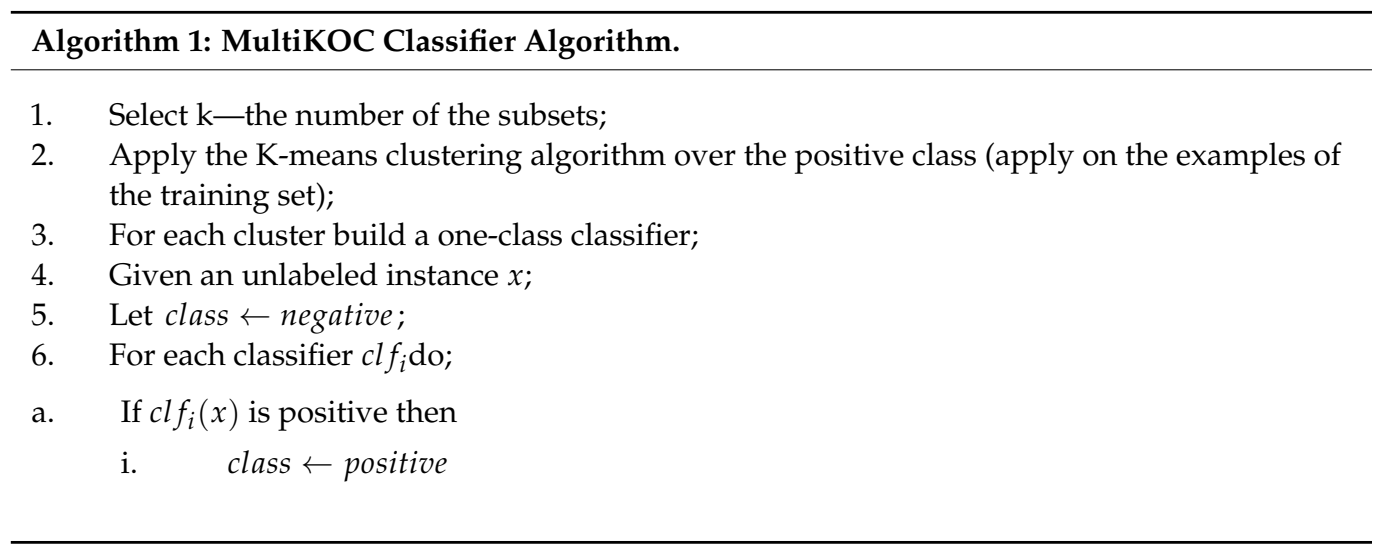




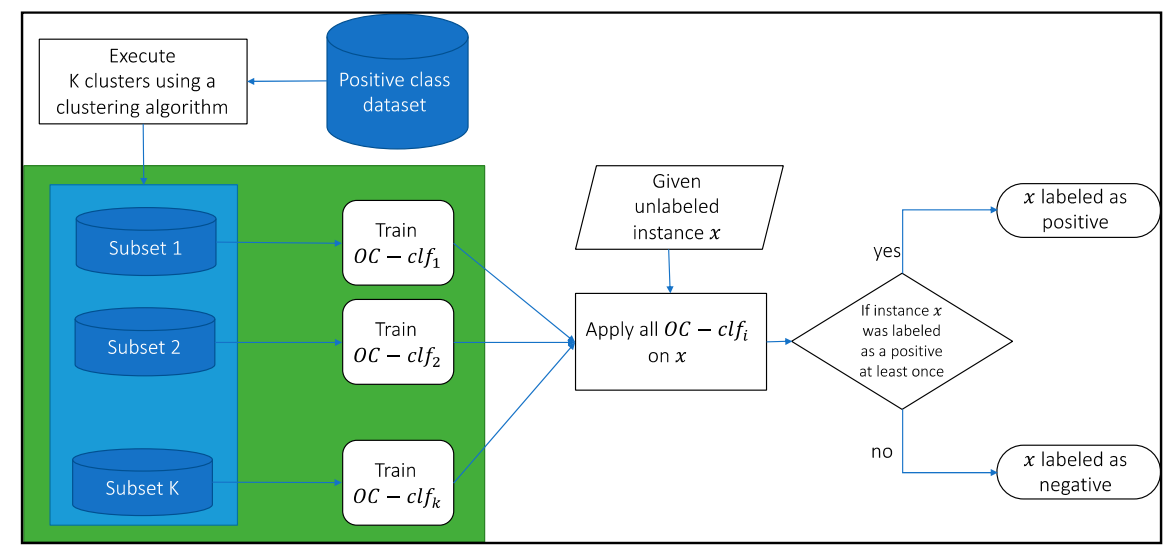

Figure 3. Illustration of the proposed method. Firstly the algorithm clusters the positive dataset into $k$ clusters, then traisn a one-class classifier for each cluster. Given a new instance, if at least one-classifier labeled it as a positive then it will be assigned as positive, otherwise negative.

It is important to note that the choice of the clustering algorithm and the number of the clusters is still a challenge. We have several proposed directions for dealing with this challenge, such as: (1) selecting the clustering algorithm to organize the data shapes; (2) using measures to evaluate the performance of the clustering; (3) using different hyperparameters to obtain the best clustering results (such as the K in K-means). However, selecting the clustering algorithm is the user choice based on the given data set.

Finally, although the proposed method uses the K-means clustering algorithm, it is different from the OC-Kmeans algorithm. In OC-Kmeans, the algorithm classifies each new instance based on its distance from the centroids of the clusters. In contrast, our method builds a classifier over each cluster, and then classifies new instances using those classifiers.

\section{Results}

We conducted experiments on three different datasets. The first dataset is syntactic which consists of two classes positive and negative samples as shown on Figure 1. Here, the data contains two classes; positive and negative of 800 samples each. The positive examples are divided into four clusters beforehand.

The second and the third data set are from the UCI repository [36]. In these data sets there are three classes. The Iris data set contains 3 classes of 50 samples each, where each class refers to a type of iris plant. The third data set is called "Thyroid gland data" which contains 150 samples from class "normal", 35 hyper, and 30 hypo class (in our experiments we assign normal as class 1 , hyper as class 2 and hypo as class 3 ).

For both data sets "Iris" and "Thyroid gland data", each time in our experiments, one-class out of the three classes was considered as the negative class, while the other two classes were considered as positive class. The generated datasets are summarized in Table 1.

Table 1. Description of the data sets. Positive and negative classess.

\begin{tabular}{ccc}
\hline Data & Positive Class & Negative Class \\
\hline SynData & blue & red \\
\hline Iris-setosa-versicolor & setosa and versicolor & verginica \\
\hline Iris-setosa-verginica & setosa and verginica & setosa \\
\hline Iris-versicolor-verginica & versicolor and verginica & class 2 and class 3 \\
\hline newthyroid class 1 & class 1 & class 3 \\
\hline class1_2_newthyroid & class 1 and class 2 & class 2 \\
\hline class1_3_newthyroid & class 1 and class 3 & class 1
\end{tabular}


In each experiment for the OC classifiers, the positive data were split into two subsetsone for training and the other for testing - while all the examples from the negative class were used for testing and not seen in training the OC. All algorithms were trained using $80 \%$ of the positive class and the remaining $20 \%$, together with all the negative examples, were used for testing. Each experiment was repeated one hundred times and the averaged results were reported.

For the two-class classifiers we considered both the positive and negative data. Similarly, the data were split into training and testing sets, where $80 \%$ was used for training and $20 \%$ for testing.

We tested the performance of MultiKOC using four different classifiers: J48, SVM, Naïve Bayes, and Random Forest versus that of the classical one-class versions of these classifiers. Additionally, we tested the MultiKOC with different values of $\mathrm{k}$ that define the number of clusters generated by K-means. We have considered $\mathrm{k}=1,2, \ldots, 6$.

The first experiment was conducted using the J48 classifier, as can be seen in Table 2. The performance of the multiKOC(J48) outperforms the classic one-class classifier.

Table 2. Results summary for J48 classifier. The column \#clusters is the optimal number of clusters obtained for MultiKOC.

\begin{tabular}{ccccc}
\hline Data & \#Clusters & MultiKOC(J48) & OC-J48 & Two-J48 \\
\hline SynData & 3 & 0.263 & 0.405 & 0.999 \\
\hline Iris-setosa-versicolor & 3 & 0.325 & 0.000 & 0.960 \\
\hline Iris-setosa-verginica & 3 & 0.303 & 0.000 & 0.956 \\
\hline Iris-versicolor-verginica & 4 & 0.286 & 0.754 & 0.995 \\
\hline newthyroid class 1 & 2 & 0.330 & 0.275 & 0.947 \\
\hline class1_2_newthyroid & 2 & 0.447 & 0.052 & 0.980 \\
\hline class1_3_newthyroid & 2 & 0.535 & 0.387 & 0.979 \\
\hline class2_3_newthyroid & 5 & 0.102 & 0.000 & 0.886 \\
\hline Average & & 0.355 & 0.267 & 0.974 \\
\hline
\end{tabular}

The second experiment's results are summarized in Table 3. The experiment was conducted using the Naïve Bayes classifier. The performance of the proposed method using the Naïve bayes classifier (i.e., multiKOC(J48)) outperforms the classic one-class Naïve Bayes classifier.

Table 3. Results summary for Naïve Bayes classifier. The column \#clusters is the optimal number of clusters obtained for MultiKOC.

\begin{tabular}{ccccc}
\hline Data & \#Clusters & MultiKOC(NB) & OC-NB & Two-NB \\
\hline SynData & 3 & 0.550 & 0.147 & 0.876 \\
\hline Iris-setosa-versicolor & 3 & 0.529 & 0.597 & 0.929 \\
\hline Iris-setosa-verginica & 3 & 0.443 & 0.271 & 0.937 \\
\hline Iris-versicolor-verginica & 4 & 0.498 & 0.963 & 1.000 \\
\hline newthyroid class 1 & 2 & 0.771 & 0.768 & 0.954 \\
\hline class1_2_newthyroid & 2 & 0.816 & 0.854 & 0.986 \\
\hline class1_3_newthyroid & 2 & 0.723 & 0.720 & 0.986 \\
\hline class2_3_newthyroid & 5 & 0.083 & 0.081 & 0.873 \\
\hline Average & & 0.619 & 0.617 & 0.952 \\
\hline
\end{tabular}


The third experiment was conducted using the Support Vector Machine classifier, as can be seen in Table 4. The performance of the multiKOC(SVM) outperforms the classic one-class SVM classifier in five experiments out of eight. Moreover, as can be seen in Table 4 , the averaged performance of the new proposed method outperforms the classical one by more than $10 \%$.

Table 4. Results summary for Support Vector Machine classifier. The column \#clusters is the optimal number of clusters obtained for MultiKOC.

\begin{tabular}{ccccc}
\hline Data & \#Clusters & MultiKOC(SVM) & OC-SVM & Two-SVM \\
\hline SynData & 3 & 0.150 & 0.157 & 1.000 \\
\hline Iris-setosa-versicolor & 3 & 0.792 & 0.691 & 0.980 \\
\hline Iris-setosa-verginica & 3 & 0.258 & 0.275 & 0.979 \\
\hline Iris-versicolor-verginica & 4 & 0.963 & 0.682 & 1.000 \\
\hline newthyroid class 1 & 2 & 0.355 & 0.242 & 0.971 \\
\hline class1_2_newthyroid & 2 & 0.676 & 0.592 & 0.925 \\
\hline class1_3_newthyroid & 2 & 0.498 & 0.302 & 0.932 \\
\hline class2_3_newthyroid & 5 & 0.082 & 0.088 & 0.944 \\
\hline Average & & 0.527 & 0.420 & 0.970 \\
\hline
\end{tabular}

The fourth experiment was conducted using the Random Forest classifier, as can be seen in Table 5. The performance of the multiKOC(RF) was equivalent to the result of the classic one-class classifier.

Table 5. Results summary for Random Forest classifier. The column \#clusters is the optimal number of clusters obtained for MultiKOC.

\begin{tabular}{ccccc}
\hline Data & \#Clusters & MultiKOC(RF) & OC-RF & Two-RF \\
\hline SynData & 3 & 0.167 & 0.357 & 0.999 \\
\hline Iris-setosa-versicolor & 3 & 0.333 & 0.312 & 0.966 \\
\hline Iris-setosa-verginica & 3 & 0.318 & 0.143 & 0.959 \\
\hline Iris-versicolor-verginica & 4 & 0.433 & 0.367 & 1.000 \\
\hline newthyroid class 1 & 2 & 0.443 & 0.422 & 0.966 \\
\hline class1_2_newthyroid & 2 & 0.610 & 0.747 & 0.987 \\
\hline class1_3_newthyroid & 2 & 0.559 & 0.478 & 0.987 \\
\hline class2_3_newthyroid & 5 & 0.085 & 0.298 & 0.900 \\
\hline Average & & 0.409 & 0.404 & 0.981 \\
\hline
\end{tabular}

Moreover, we can see that in all the algorithms above, the proposed MultiKOC methods outperform or are comparable to the existing methods. The results are summarized in Table 6.

Table 6. Results summary for all the classifiers from the different datasets.

\begin{tabular}{ccc}
\hline Classifier & MultiKOC & OC \\
\hline J48 & 0.355 & 0.267 \\
\hline Naïve Bayes & 0.619 & 0.617 \\
\hline Support Vector Machine & 0.527 & 0.420 \\
\hline Random Forest & 0.409 & 0.404 \\
\hline
\end{tabular}


Another experiment was conducted to check the effectiveness of the number of the clusters on the performance of the MultiKOC as can be seen in Tables 7-9 for each dataset.

Table 7. Results summary for MultiKOC method with 2, 3, 4, 5 clusters on Synthetic data.

\begin{tabular}{ccccc}
\hline Classifier & $\mathbf{2}$ & $\mathbf{3}$ & $\mathbf{4}$ & $\mathbf{5}$ \\
\hline MultiKOC(J48) & 0.233 & 0.263 & 0.167 & 0.167 \\
\hline MultiKOC(SVM) & 0.148 & 0.150 & 0.153 & 0.158 \\
\hline MultiKOC(NB) & 0.186 & 0.550 & 0.514 & 0.337 \\
\hline MultiKOC(RF) & 0.167 & 0.167 & 0.167 & 0.167 \\
\hline
\end{tabular}

Table 8. Results summary for MultiKOC method with 2, 3, 4, 5 clusters on Iris-setosa-versicolor data.

\begin{tabular}{ccccc}
\hline Classifier & $\mathbf{2}$ & $\mathbf{3}$ & $\mathbf{4}$ & $\mathbf{5}$ \\
\hline MultiKOC(J48) & 0.344 & 0.325 & 0.290 & 0.272 \\
\hline MultiKOC(SVM) & 0.770 & 0.792 & 0.679 & 0.695 \\
\hline MultiKOC(NB) & 0.388 & 0.529 & 0.547 & 0.379 \\
\hline MultiKOC(RF) & 0.333 & 0.333 & 0.419 & 0.371 \\
\hline
\end{tabular}

Table 9. Results summary for MultiKOC method with 2, 3, 4, 5 clusters on new thyroid class 1 data.

\begin{tabular}{ccccc}
\hline Classifier & $\mathbf{2}$ & $\mathbf{3}$ & $\mathbf{4}$ & $\mathbf{5}$ \\
\hline MultiKOC(J48) & 0.356 & 0.330 & 0.316 & 0.316 \\
\hline MultiKOC(SVM) & 0.369 & 0.355 & 0.347 & 0.342 \\
\hline MultiKOC(NB) & 0.505 & 0.771 & 0.437 & 0.490 \\
\hline MultiKOC(RF) & 0.385 & 0.443 & 0.373 & 0.347 \\
\hline
\end{tabular}

In conclusion, in general, the performance of MultiKOC algorithm does not depend in the number of the clusters. There are few cases that the performance of some classifiers was affected by the number of clusters, as a result, our future work will be focus on this issue.

\section{Discussion}

This study suggests MultiKOC, a novel approach for performing one-class classification that is based on partitioning the training data into clusters to model each cluster by the one-class model.

The current results show that it is possible to build up a multi-one-class classifier with a combined clustering beforehand process based only on positive examples yielding a significant improvement over the one-class and similar results as the two-class. However, the MultiKOC would include more interpretable classifiers than the two-class version as one can perform a deep analysis to explore the hidden structure of the data. Additionally, MultiKOC is robust at dealing with outliering examples, while the one-class version might add more clusters to capture those outliers and reduce their influence on the performance of the classifications.

Further research could proceed in several interesting directions. First, the suitability of the framework of our approach for different data types could be investigated. Second, it would be interesting to apply our approach to other types of classifiers and to more robust clustering methods such as Mean-Shift [37].

In the current version of MultiKOC we have considered only a one-class algorithm. One future approach is to perform an ensemble of OC and suggest a suitable voting procedure to assign the label to the new unlabeled instance. 
Author Contributions: L.A., M.B., W.K. and M.Y. have contributed equally to this study in all its parts: conceptualization; methodology; software; validation; formal analysis; investigation; resources; data curation; writing — original draft preparation; writing_-review and editing; visualization; supervision; project administration; funding acquisition. All authors have read and agreed to the published version of the manuscript.

Funding: This research received no external funding.

Data Availability Statement: We used the Iris dataset from the UCI repository and the synthetic dataset that was constructed as described in the Experiments section.

Conflicts of Interest: The authors declare no conflict of interest.

\section{References}

1. Kowalczyk, A.; Raskutti, B. One Class SVM for Yeast Regulation Prediction. SIGKDD Explor. 2002, 4, 99-100. [CrossRef]

2. Spinosa, E.J.; de Carvalho, A.C.P.L.F. Support Vector Machines for Novel Class Detection in Bioinformatics. Genet. Mol. Res. 2005, 4, 608-615. [PubMed]

3. Crammer, K.; Chechik, G. A Needle in a Haystack: Local One-Class Optimization. In Proceedings of the 21st International Conference on Machine Learning (ICML-04), Banff, AB, Canada, 4-8 July 2004.

4. Gupta, G.; Ghosh, J. Robust One-Class Clustering Using Hybrid Global and Local Search; ACM Press: New York, NY, USA, 2005.

5. Yousef, M.; Najami, N.; Khalifa, W. A Comparison Study Between One-Class and Two-Class Machine Learning for MicroRNA Target Detection. J. Biomed. Sci. Eng. 2010, 3, 247-252. [CrossRef]

6. Tarassenko, L. Novelty Detection for the Identification of Masses in Mammograms. In Proceedings of the 1995 th International Conference on Artificial Neural Networks, Cambridge, UK, 26-28 June 1995; IEEE: Cambridge, UK, 1995; Volume 1995, pp. 442-447.

7. Costa, M.; Moura, L. Automatic Assessment of Scintmammographic Images Using a Novelty Filter. In Proceedings of the Annual Symposium on Computer Application in Medical Care, New York, NY, USA, 28 October-1 November 1995; pp. 537-541.

8. Reyes, J.A.; Gilbert, D. Prediction of Protein-Protein Interactions Using One-Class Classification Methods and Integrating Diverse Biological Data. J. Integr. Bioinform. 2007, 4, 208-223. [CrossRef]

9. Manevitz, L.M.; Yousef, M. One-Class Svms for Document Classification. J. Mach. Learn. Res. 2002, 2, 139-154.

10. Thirion, B.; Faugeras, O. Feature Characterization in FMRI Data: The Information Bottleneck Approach. Med. Image Anal. 2004, 8 , 403-419. [CrossRef] [PubMed]

11. Koppel, M. Authorship Verification as a One-Class Classification Problem. In Proceedings of the Twenty-First International Conference on Machine Learning, Banff, AB, Canada, 4-8 July 2004; ACM Press: Banff, AB, Canada, $2004 ;$ p. 62.

12. Gonzalez-Soler, L.J.; Gomez-Barrero, M.; Chang, L.; Suarez, A.P.; Busch, C. On the Impact of Different Fabrication Materials on Fingerprint Presentation Attack Detection. In Proceedings of the 2019 International Conference on Biometrics (ICB), Crete, Greece, 4-7 June 2019; IEEE: Crete, Greece, 2019; pp. 1-6.

13. Yousef, M.; Jung, S.; Showe, L.C.; Showe, M.K. Learning from Positive Examples When the Negative Class Is UndeterminedMicroRNA Gene Identification. Algorithms Mol. Biol. 2008, 3. [CrossRef] [PubMed]

14. Yousef, M.; Allmer, J.; Khalifa, W. Sequence Motif-Based One-Class Classifiers Can Achieve Comparabl e Accuracy to Two-Class Learners for Plant MicroRNA Detection. J. Biomed. Sci. Eng. 2015. [CrossRef]

15. Yousef, M.; Khalifa, W.; AbedAllah, L. Ensemble Clustering Classification Compete SVM and One-Class Classifiers Applied on Plant MicroRNAs Data. J. Integr. Bioinform. 2016, 13, 304. [CrossRef] [PubMed]

16. Yousef, M.; Khalifa, W. A Zero-Norm Feature Selection Method for Improving the Performance of the One-Class Machine Learning for MicroRNA Target Detection. In Proceedings of the 5th International Symposium on Health Informatics and Bioinformatics (HIBIT), Ankara, Turkey, 20-22 April 2010; pp. 45-50. [CrossRef]

17. You, L.; Peng, Q.; Xiong, Z.; He, D.; Qiu, M.; Zhang, X. Integrating Aspect Analysis and Local Outlier Factor for Intelligent Review Spam Detection. Future Gener. Comput. Syst. 2020, 102, 163-172. [CrossRef]

18. AbedAllah, L.; Shimshoni, I. k Nearest Neighbor Using Ensemble Clustering. In Data Warehousing and Knowledge Discovery: Proceedings of the 14th International Conference, DaWaK 2012, Vienna, Austria, 3-6 September 2012; Cuzzocrea, A., Dayal, U., Eds.; Springer: Berlin/Heidelberg, Germany, 2012; pp. 265-278. ISBN 978-3-642-32584-7.

19. Landgrebea, T.C.; Paclıka, D.M.T.P.; Andrew, R.P.D.C.M. One-Class and Multi-Class Classifier Combining for Ill-Defined Problems; CiteSeer: University Park, PA, USA, 2005.

20. Lai, C.; Tax, D.M.; Duin, R.P.; Pękalska, E.; Paclík, P. On Combining One-Class Classifiers for Image Database Retrieval. In International Workshop on Multiple Classifier Systems; Springer: Berlin/Heidelberg, Germany, 2002; pp. $212-221$.

21. Tax, D.M.; Duin, R.P. Combining One-Class Classifiers. In International Workshop on Multiple Classifier Systems; Springer: Berlin/Heidelberg, Germany, 2001; pp. 299-308.

22. Lyu, S.; Farid, H. Steganalysis Using Color Wavelet Statistics and One-Class Support Vector Machines; SPIE: Bellingham, WA, USA, 2004; Volume 5306, pp. 35-45. 
23. Menahem, E.; Rokach, L.; Elovici, Y. Combining One-Class Classifiers via Meta Learning. In Proceedings of the 22nd ACM international conference on Information \& Knowledge Management, San Francisco, CA, USA, 26 December 2013; pp. 2435-2440.

24. Ban, T.; Abe, S. Implementing Multi-Class Classifiers by One-Class Classification Methods. In Proceedings of the 2006 IEEE International Joint Conference on Neural Network Proceedings, Vancouver, BC, Canada, 16-21 July 2006; pp. 327-332.

25. Spinosa, E.J.; de Carvalho, A.C. Combining One-Class Classifiers for Robust Novelty Detection in Gene Expression Data. In Brazilian Symposium on Bioinformatics; Springer: Berlin/Heidelberg, Germany, 2005; pp. 54-64.

26. Zhang, J.; Lu, J.; Zhang, G. Combining One Class Classification Models for Avian Influenza Outbreaks. In Proceedings of the 2011 IEEE Symposium on Computational Intelligence in Multicriteria Decision-Making (MDCM), Paris, France, 11-15 April 2011; pp. 190-196.

27. Tax, D.M.J. One-Class Classification; Concept-Learning in the Absence of Counter-Examples. Ph.D. Thesis, Technische Universiteit Delft, Delft, The Netherlands, 2001.

28. Schölkopf, B.; Platt, J.C.; Shawe-Taylor, J.; Smola, A.J.; Williamson, R.C. Estimating the Support of a High-Dimensional Distribution. Neural Comput. 2001, 13, 1443-1471. [CrossRef] [PubMed]

29. Chang, C.-C.; Lin, C.-J. LIBSVM: A Library for Support Vector Machines. ACM Trans. Intell. Syst. Technol. 2011, 2, 1-27. [CrossRef]

30. Witten, I.H.; Frank, E.; Hall, M.A. Introduction to Weka. In Data Mining: Practical Machine Learning Tools and Techniques; Elsevier: Amsterdam, The Netherlands, 2011; pp. 403-406.

31. Michael, R.B.; Cebron, N.; Dill, F.; Thomas, R.G.; Kotter, T.; Meinl, T.; Ohl, P.; Sieb, C.; Thiel, K.; Wiswedel, B. KNIME: The Konstanz Information Miner. Studies in Classification, Data Analysis, and Knowledge Organization (GfKL 2007); Springer: Berlin/Heidelberg, Germany, 2007; pp. 1431-8814.

32. Schölkopf, B.; Burges, C.J.C.; Smola, A.J. Advances in Kernel Methods; MIT Press: Cambridge, MA, USA, 1999.

33. Sain, S.R.; Vapnik, V.N. The Nature of Statistical Learning Theory. Technometrics 1996. [CrossRef]

34. Krawczyk, B.; Woźniak, M.; Cyganek, B. Clustering-Based Ensembles for One-Class Classification. Inf. Sci. 2014, 264, 182-195. [CrossRef]

35. Hempstalk, K.; Frank, E.; Witten, I.H. One-Class Classification by Combining Density and Class Probability Estimation. In Machine Learning and Knowledge Discovery in Databases; Lecture Notes in Computer Science; Daelemans, W., Goethals, B., Morik, K., Eds.; Springer: Berlin/Heidelberg, Germany, 2008; Volume 5211, pp. 505-519. ISBN 978-3-540-87478-2.

36. Dua, D.; Graff, C. UCI Machine Learning Repository; University of California Irvine, School of Information and Computer Sciences: Irvine, CA, USA, 2007.

37. Comaniciu, D.; Meer, P. Mean Shift: A Robust Approach toward Feature Space Analysis. IEEE Trans. Pattern Anal. Mach. Intell. 2002, 24, 603-619. [CrossRef] 